\title{
Transient Pc3 wave activity generated by a hot flow anomaly: Cluster, Rosetta, and ground-based observations
}

\author{
J. P. Eastwood, ${ }^{1}$ S. J. Schwartz, ${ }^{1}$ T. S. Horbury, ${ }^{1}$ C. M. Carr, ${ }^{1}$ K.-H. Glassmeier, ${ }^{2,3}$ \\ I. Richter, ${ }^{2}$ C. Koenders, ${ }^{2}$ F. Plaschke, ${ }^{2}$ and J. A. Wild ${ }^{4}$ \\ Received 11 January 2011; revised 4 May 2011; accepted 10 May 2011; published 27 August 2011.
}

[1] Pc3 pulsations are observed in the magnetosphere with wave periods of 10-45 s. Two distinct populations have been observed; one exhibits a frequency dependence on the solar wind magnetic field strength, whereas the other does not. The first population is explained in terms of a model where the bow shock reflects ions which generate upstream foreshock ULF waves. These waves are convected through the shock to the dayside magnetopause and thus to the magnetosphere. The source of the second population is not well understood. In this paper we examine the generation of a transient patch of Pc3 wave activity due to a hot flow anomaly (HFA) using a unique spacecraft conjunction that occurred during the first Earth flyby of the Rosetta spacecraft. Cluster, upstream of the bow shock and close to the Sun-Earth line observed an HFA. At this time Rosetta was nearing closest approach and together with ground magnetometer stations, observed a transient interval of Pc3 wave activity. Analysis also shows that the Pc3 waves occurred in the absence of a ULF wavefield just upstream of the bow shock. This result shows that HFAs can be a source of Pc3 wave activity, and may explain in part the origin of the second population of Pc3 waves. It also demonstrates in new detail the manner in which kinetic physics at the bow shock, driven by structure in the solar wind, can influence magnetospheric dynamics.

Citation: Eastwood, J. P., S. J. Schwartz, T. S. Horbury, C. M. Carr, K.-H. Glassmeier, I. Richter, C. Koenders, F. Plaschke, and J. A. Wild (2011), Transient Pc3 wave activity generated by a hot flow anomaly: Cluster, Rosetta, and ground-based observations, J. Geophys. Res., 116, A08224, doi:10.1029/2011JA016467.

\section{Introduction}

[2] Pc3 waves, which have periods of the order of $10-45 \mathrm{~s}$, are commonly observed in the magnetosphere [Hughes, 1994]. Two distinct populations of Pc3 waves have been identified [Gul'elmi, 1974; Slawinski et al., 1988]. The first population exhibits a frequency dependence on the solar wind magnetic field strength $|\mathrm{B}|_{\mathrm{sw}}$. The second population of Pc3 waves has a peak frequency between $20-30 \mathrm{mHz}$ and does not appear to depend on $|\mathrm{B}|_{\mathrm{sw}}$. This points to the existence of multiple generation mechanisms.

[3] Since Pc3 waves are found to occur more often when the IMF is radial [Troitskaya et al., 1972, and references therein], the source of the first Pc3 population is interpreted in terms of a model where under radial IMF conditions, the foreshock ULF wavefield sits directly upstream of the bow

\footnotetext{
${ }^{1}$ Blackett Laboratory, Imperial College London, London, UK.

${ }^{2}$ IGEP, Technische Universität Braunschweig, Braunschweig, Germany.

${ }^{3}$ Also at Max Planck Institute for Solar System Research, KatlenburgLindau, Germany.

${ }^{4}$ Space Plasma Environment and Radio Science Group, Department of Physics, Lancaster University, Lancaster, UK.

Copyright 2011 by the American Geophysical Union. 0148-0227/11/2011JA016467
}

shock. The foreshock is the region of the solar wind magnetically connected to the bow shock [e.g., Eastwood et al., 2005b]. It is filled with backstreaming particles [e.g., Fuselier, 1994, 1995] and a number of different plasma instabilities have been observed, which leads to the generation of different waves [e.g., Le and Russell, 1994; Burgess, 1995, 1997]. Backstreaming beams of ions generate ultralow-frequency (ULF) waves with periods of $\sim 30 \mathrm{~s}$ in the spacecraft frame [Eastwood et al., 2005a]. Although the foreshock ULF waves are moving upstream in the solar wind rest frame, the supersonic solar wind flow convects the waves into the magnetosheath and to the magnetopause, where ultimately the waves mode convert to Alfvenic fluctuations whose frequencies are governed by eigenmode resonances [Clausen et al., 2009]. Extensive observations [Takahashi et al., 1984; Odera, 1986; Le and Russell, 1994; Chi and Russell, 1998; Clausen et al., 2009] connecting the upstream wave measurements to the ground have been published. This model predicts that Pc3 waves tend to be generated when the solar wind magnetic field is radial and that $\mathrm{f}_{\mathrm{Pc} 3}$, the frequency of the subsequently observed waves, depends on $|\mathrm{B}|_{\mathrm{sw}}$ [Gul'elmi, 1974; Takahashi et al., 1984; Odera, 1986]. Initial analysis suggested that $\mathrm{f}_{\mathrm{Pc} 3} \sim 6 \mathrm{IBI}_{\mathrm{sw}} \mathrm{mHz}$ [Gul'elmi, 1974], where $|\mathrm{B}|_{\mathrm{sw}}$ is measured in nanotesla, but other models accounting for the magnetic field cone angle $\theta_{\mathrm{Bx}}$ have 
also been proposed, e.g., $\left.\mathrm{f}_{\mathrm{Pc} 3} \sim 7.68 \mathrm{IB}\right|_{\mathrm{sw}} \cos ^{2} \theta_{\mathrm{Bx}} \mathrm{mHz}$ [Takahashi et al., 1984].

[4] The source of the second population is not well understood. Slawinski et al. [1988] speculated on other causes of Pc3 waves, suggesting that a variety of phenomena could be responsible, including magnetopause surface wave excitations, flux transfer events, and ionospheric processes. Gul'elmi [1974] speculated on the possibility of their generation being due to a source interior to the magnetosphere. One line of evidence for the possibility of other generation mechanisms comes from a statistical study that associated Pc3 waves with Magnetic Impulse Events (MIEs) [Shields et al., 2003]. MIEs [Lanzerotti et al., 1986] are transient disturbances of the magnetic field at high latitudes, lasting a few minutes. When measured by several magnetometer stations, the structure of the disturbance can be examined; the majority of MIEs are in fact Traveling Current Vortices (TCVs) [Zesta et al., 2002] (also known as Traveling Convection Vortices [see Glassmeier, 1992]). One important source of MIEs and TCVs is hot flow anomalies (HFAs) [Sibeck et al., 1999; Eastwood et al., 2008; Jacobsen et al., 2009].

[5] HFAs are a kinetic-physics phenomenon intrinsic to the bow shock and depend on the existence of current sheets in the solar wind [Schwartz, 1995; Schwartz et al., 2000]. If the solar wind convection electric field points into the current sheet on at least one side [Thomsen et al., 1993] then reflected ions at the shock are channeled into the discontinuity, resulting in a hot plasma population that significantly disrupts the solar wind flow [Lucek et al., 2004; Omidi and Sibeck, 2007; Eastwood et al., 2008]. This can result in dramatic motion of the magnetopause [Sibeck et al., 1999], with associated magnetospheric disturbances [Sitar et al., 1998; Weatherwax et al., 1999; Fillingim et al., 2011]. In their study, Shields et al. [2003] demonstrated the close association of Pc3 waves and MIEs, but the subsequent association with HFAs remains unclear.

[6] In this paper we present new observations that show the generation of Pc3 waves as a result of an HFA. What is particularly unusual is that during this event, Cluster, just upstream of the nose of the bow shock, did not see any foreshock waves that could reasonably be concluded to be the direct source of the Pc3 wave activity. The generation of the observed magnetospheric Pc3 waves thus relies on kinetic physics effects associated with transient solar wind structure - namely an HFA - and demonstrates that intervals of Pc3 waves can be generated in the absence of a significant ULF wavefield just upstream of the bow shock.

\section{Data}

[7] In this paper we use data from the Cluster [Escoubet et al., 2001] and Rosetta [Glassmeier et al., 2007a] spacecraft, as well as ground based magnetometer data from several different networks. This event occurred on 4 March 2005, during the first Rosetta Earth flyby.

\subsection{Cluster}

[8] The four Cluster spacecraft were launched in 2000 into synchronized polar orbits [Escoubet et al., 2001]. During the first few years of the mission, the orbits were maintained so that the four spacecraft formed a regular tetrahedron at apogee $\left(19.6 \mathrm{R}_{\mathrm{E}}\right)$. The scale size of the tetrahedron was varied periodically (approximately every 6 or 12 months). During the first part of 2005, the tetrahedron scale size was $\sim 1000 \mathrm{~km}$. In this study, data from the Fluxgate Magnetometer (FGM) experiment [Balogh et al., 2001] and the Cluster Ion Spectrometry (CIS) experiment [Rème et al., $2001]$ is used. The magnetic field data is shown at high resolution $(22.2$ vectors $/ \mathrm{s})$ and the moments of the ion plasma distribution are shown at $4 \mathrm{~s}$ resolution.

\subsection{Rosetta}

[9] The main target of the ESA Rosetta mission is the comet 67/P Churyumov-Gerasimenko [Glassmeier et al., 2007a]. Rosetta is due to rendezvous with the comet in 2014. Since its launch in 2004, it has executed three Earth flybys in addition to one Mars flyby and flybys of the asteroids 2867 Steins and 21 Lutetia. The first Earth flyby occurred on 4 March 2005, which presented an opportunity to perform serendipitous science observations. Rosetta approached the Earth from the deep tail, and reached closest approach at an altitude of $1961 \mathrm{~km}$ at 22:00 UT.

[10] We have used data from the Fluxgate Magnetometer (RPC-MAG) [Glassmeier et al., 2007b] which forms part of the Rosetta Plasma Consortium [Carr et al., 2007]. Magnetometer data is available for several days before and after the flyby. The magnetic field data is shown at $1 \mathrm{~s}$ resolution, and has been processed to remove any spacecraft sources of noise. Data from the Ion-Electron Sensor (RPC-IES) was also surveyed [Burch et al., 2007], but in preparation for the flyby the instrument had been turned off prior to the events reported here and so only the Rosetta magnetic field data has been analyzed.

\subsection{Ground-Based Magnetometer Data}

[11] Ground-based magnetic field measurements have been provided by the UK sub-auroral magnetometer network (SAMNET) [Davison and Orr, 1989]. This network provides measurements at 13 stations stretching from Russia in the east to Iceland in the west, and from the southern UK to northern Finland. It incorporates data from several magnetometers operated by the British Geological Survey of the International Monitor for Auroral Geomagnetic Effects (IMAGE) array [Lühr et al., 1998]. Data from these magnetometers, usually archived at $\sim 10 \mathrm{~s}$ resolution, are archived at $1 \mathrm{~s}$ resolution to match the data resolution from the SAMNET stations. Following subtraction of the stationspecific quiet-day trend, magnetic field vectors are presented in an HDZ coordinate system, where the $\mathrm{H}$ direction points toward average magnetic north at the site, with $\mathrm{Z}$ vertically downward and $\mathrm{D}$ making up the right-handed set.

[12] Data has also been used from the THEMIS ground based array [Mende et al., 2008; Russell et al., 2008]. While this event occurred before the beginning of the THEMIS mission in 2007, ground magnetometer data was already available from certain sites. In particular, we have used data from the Petersburg site (PTRS) which is also one of the THEMIS Education and Public Outreach magnetometer sites [Peticolas et al., 2008]. Again, data are shown with $\mathrm{H}$ aligned to magnetic north, $\mathrm{D}$ to magnetic east and $\mathrm{Z}$ pointing vertically down. The time resolution of the data is 2 vectors/s, and the daily mean has again been removed. 
Table 1. Geographic Locations of Magnetometer Stations Used in this Analysis

\begin{tabular}{lcccc}
\hline \multicolumn{1}{c}{ Station } & Code & $\begin{array}{c}\text { Magnetic } \\
\text { Latitude }\end{array}$ & $\begin{array}{c}\text { Geographic } \\
\text { Latitude }\end{array}$ & $\begin{array}{c}\text { Geographic } \\
\text { Longitude }\end{array}$ \\
\hline Petersburg & PTRS & 60.3 & 56.83 & 226.84 \\
Kilpisjärvi & KIL & 65.9 & 69.02 & 20.79 \\
Hella & HLL & 64.7 & 63.77 & -20.56 \\
Oulujärvi & OUJ & 61.0 & 64.52 & 27.23 \\
Faroes & FAR & 61.0 & 62.05 & -7.02 \\
Hankasalmi & HAN & 58.7 & 62.25 & 26.60 \\
Lerwick & LER & 58.3 & 60.13 & -1.18 \\
Nurmijärvi & NUR & 57.0 & 60.50 & 24.65 \\
Uppsala & UPS & 56.7 & 59.90 & 17.35 \\
Crooktree & CRK & 55.2 & 57.09 & -2.64 \\
Borok & BOR & 54.2 & 58.03 & 38.33 \\
Eskdalemuir & ESK & 53.3 & 55.32 & -3.20 \\
York & YOR & 51.3 & 53.95 & -1.05 \\
Hartland & HAD & 48.4 & 50.99 & -4.48 \\
\hline
\end{tabular}

[13] Details of all the ground based magnetometer data used in this analysis are shown in Table 1.

\section{Observations}

\subsection{Cluster HFA Observations}

[14] Figure 1 shows magnetic field and ion plasma data observed by Cluster on 4 March 2005 between 21:00 21:20 UT. During this time Cluster was in the solar wind on the outbound leg of its orbit, having crossed the bow shock at approximately 18:45 UT. The average solar wind speed and density were stable in the two hours surrounding this event (20:00-22:00 UT).

[15] Just after 21:09 UT, Cluster observed a dramatic reduction in the plasma density, together with an increase in the plasma temperature and a deflection in the bulk plasma velocity. Associated with this drop in the plasma density was a drop in the magnetic field strength. Bounding this region on the trailing edge is an enhanced region of magnetic field. These signatures are characteristic of a hot flow anomaly (HFA) [Schwartz et al., 2000; Omidi and Sibeck, 2007; Eastwood et al., 2008; Jacobsen et al., 2009]. An unusual feature of this hot flow anomaly is the structure of the leading edge, which consists of a small packet of compressive waves. These waves are decorrelated between the spacecraft, and their period is of the order of a few seconds; the period at each spacecraft is different. Minimum variance analysis shows that the waves are right handed in the spacecraft frame, approximately circularly polarized, and propagating at an angle of $\sim 30^{\circ}$ to the magnetic field. These waves are a unique feature of this HFA and it will be shown below that they are in fact unrelated to the Pc3 wave activity seen inside the magnetosphere.

[16] The majority of foreshock ULF waves with periods of $\sim 30 \mathrm{~s}$ are left-handed in the spacecraft frame as they are produced by sunward propagating foreshock ion beams via the right hand resonant ion beam instability, but then convected by the solar wind in a direction opposite to their propagation. When waves that are right-handed in the spacecraft frame are observed, this either indicates the presence of intrinsically right-handed (fast mode) waves propagating in the direction of the solar wind flow, or lefthanded (Alfven/ion cyclotron) waves propagating sunward [Eastwood et al., 2003]. Because the waves are decorrelated between the spacecraft it is not possible to determine the precise nature of the wave propagation from multipoint analysis. However, their frequency is several times higher than the $30 \mathrm{~s}$ waves that are typically observed in the foreshock. No foreshock ULF waves (with the characteristic $30 \mathrm{~s}$ periods) were observed in the intervals surrounding the HFA.

[17] Although multispacecraft analysis can in principle be used to determine the orientation of boundaries associated with a HFA [Lucek et al., 2004], in this event the Cluster spacecraft separation was relatively large $(>1000 \mathrm{~km})$. This meant that a timing analysis was not possible in this particular event. As mentioned above, the wave packet on the leading edge is decorrelated between the spacecraft, and the additional fact that the waves are likely to be propagating at a different speed and direction to the underlying leading edge, means that the orientation of this structure cannot be determined from the wave packet.

[18] To determine the orientation of the underlying discontinuity, we use coplanarity assuming that the underlying discontinuity is tangential in nature. It is thought that HFAs are primarily formed by the interaction of TDs with the bow shock [Schwartz, 1995], and multipoint analysis using Cluster data has in fact shown that the majority of discontinuities in the solar wind are tangential [Knetter et al., 2004].

[19] The intervals chosen for the upstream and downstream magnetic field are shown in Figure 1. Between 21:06 and 21:07 UT, $\mathbf{B}_{\mathbf{1}}=\left[\begin{array}{l}1.28 \\ 0.662 .75\end{array}\right] \mathrm{nT}$ and between $21: 10$ and $21: 11 \mathrm{UT}, \mathbf{B}_{\mathbf{2}}=\left[\begin{array}{lll}0.42 & 1.16 & 3.00\end{array}\right] \mathrm{nT}$. This leads to a normal direction $\mathbf{n}=\left(\mathbf{B}_{\mathbf{1}} \times \mathbf{B}_{\mathbf{2}}\right) /\left|\mathbf{B}_{\mathbf{1}} \times \mathbf{B}_{\mathbf{2}}\right|=[-0.38-0.840 .38] . \mathrm{A}$ key requirement for the formation of a HFA is that the solar wind convection electric field points toward the discontinuity from at least one side [Thomsen et al., 1993; Schwartz et al., 2000, Figure 1]. To test this, we note that in this interval the overall solar wind velocity is stable at $-400 \mathrm{~km} \mathrm{~s}^{-1}$ in the $\mathrm{x}_{\mathrm{GSE}}$ direction. Since $\mathbf{n} . \mathbf{v}_{\mathbf{s w}}$ is positive, this means that before the HFA was observed, the convection electric field should be anti-parallel to the normal direction; that is $\mathbf{E}_{\text {conv, } \mathbf{1}} \cdot \mathbf{n}$ should be negative. To visualize this, note that the underlying discontinuity is moving toward the Earth with its normal oriented toward the Earth $\left(\mathrm{n}_{\mathrm{x}}<0\right)$. As such, the convection electric field before the arrival of the TD should have a component pointing sunward, antiparallel to the normal. After the HFA was observed, the convection electric field should be parallel to the normal, and therefore $\mathbf{E}_{\mathbf{c o n v}, \mathbf{2}} \cdot \mathbf{n}$ should be positive. In the upstream interval, $\mathbf{E}_{\mathbf{c o n v}, \mathbf{1} \cdot \mathbf{n}}=1.03 \mathrm{mVm}^{-1}$; downstream $\mathbf{E}_{\mathbf{c o n v}, \mathbf{2} \cdot \mathbf{n}}=$ $1.19 \mathrm{mVm}^{-1}$. Therefore the first key criterion for HFA formation is met, since the convection electric field is appropriately oriented after the HFA is observed, on the downstream side.

[20] The second criterion for HFA formation is that the magnetic field is connected to the shock on at least one side of the discontinuity [Omidi and Sibeck, 2007]. This requirement ensures that once the TD touches the shock, a population of backstreaming particles exists that can be channeled into the discontinuity and can ultimately disrupt the solar wind flow. To test this, the magnetic field was traced from the location of Cluster to the location of a model bow shock and $\theta_{\mathrm{Bn}}$, the angle between the magnetic field and the local shock normal, was calculated. The shock location was calculated using the Farris et al. [1991] model, 
Cluster 2005-03-04
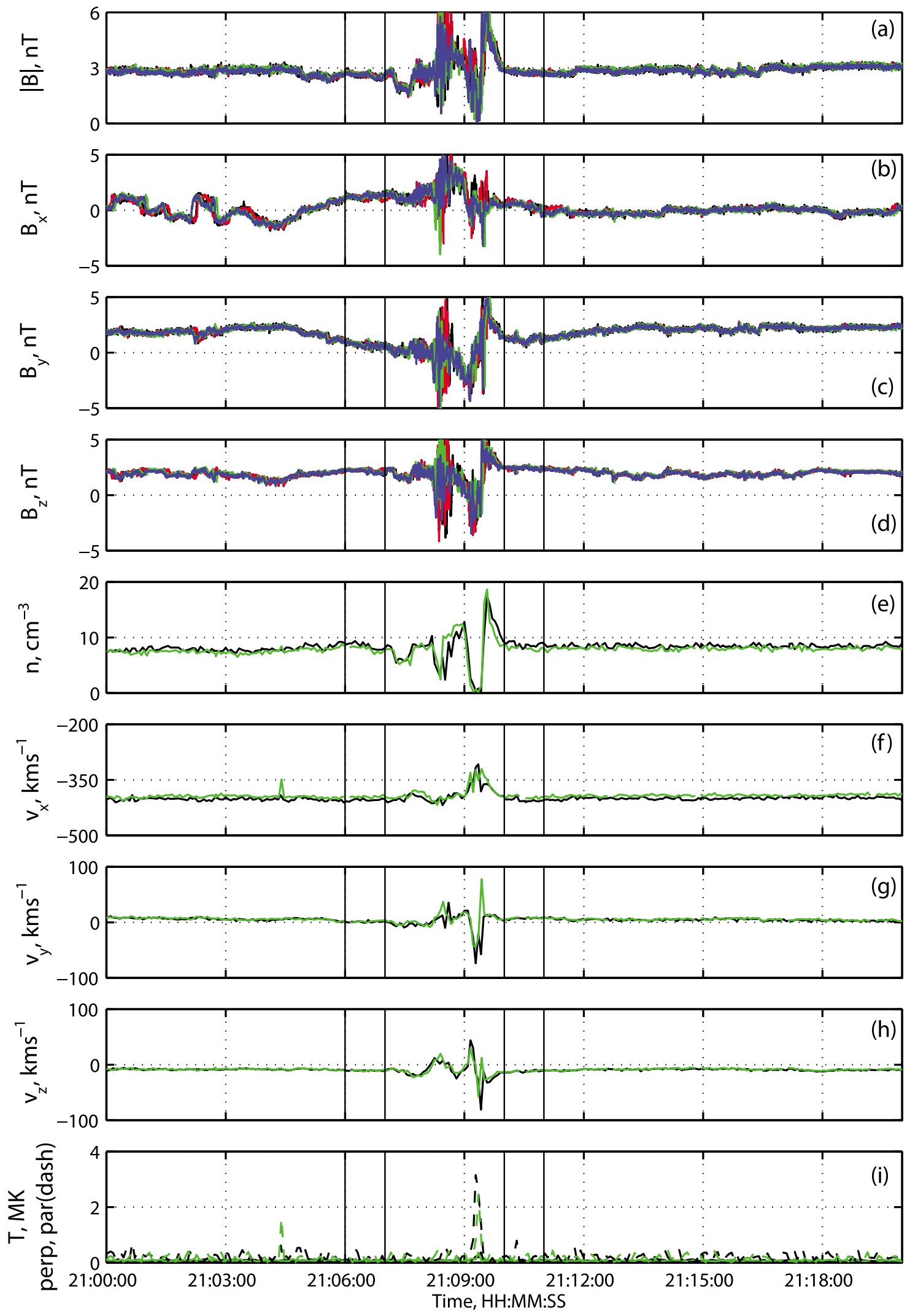

Figure 1 

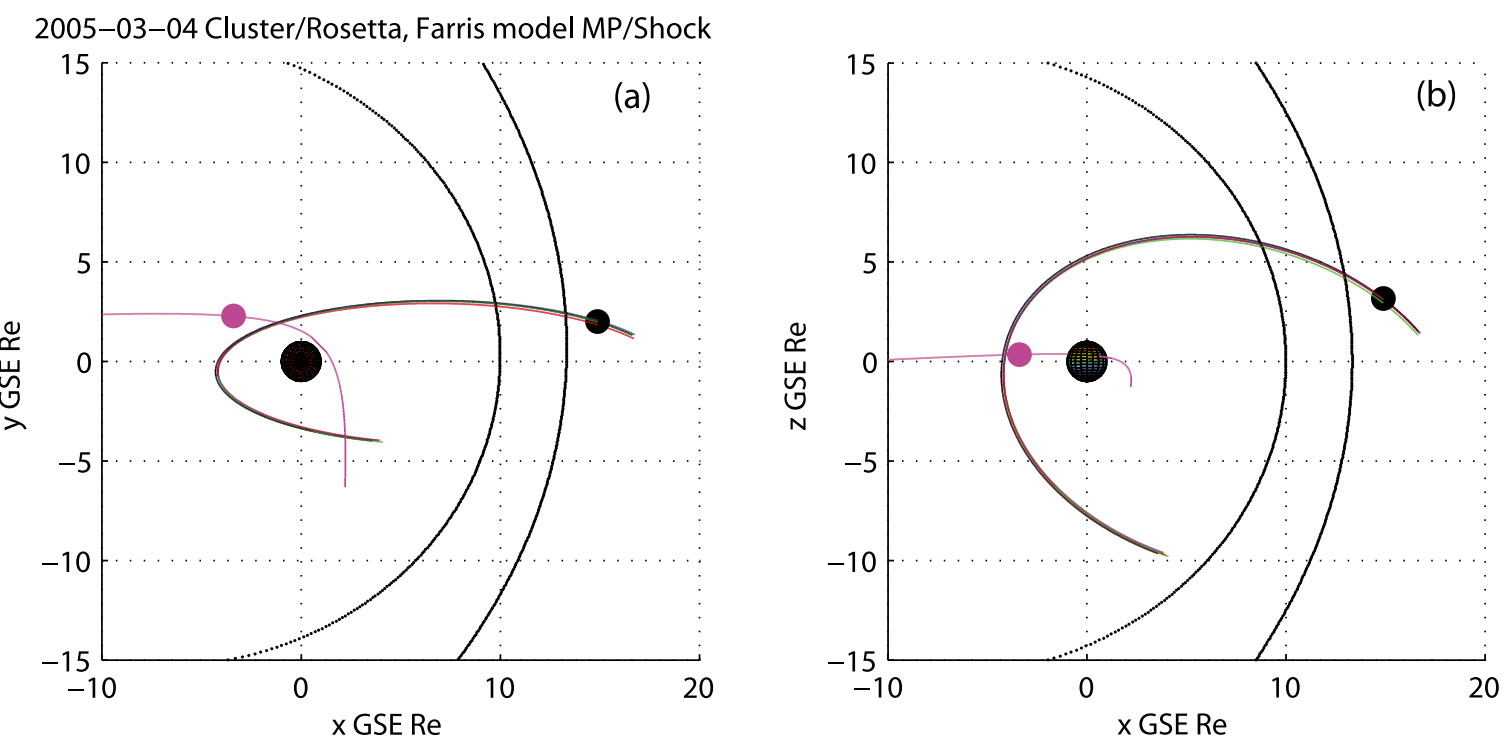

Figure 2. Location of the Cluster and Rosetta spacecraft (a) projection into the $x-y$ GSE plane (b) projection into the $x-z$ GSE plane. Cluster was upstream of the bow shock on the outbound leg of its orbit. The Farris et al. model shock and magnetopause are shown, appropriately scaled to the observed solar wind conditions. The Rosetta orbit and location is shown in magenta. Rosetta approached the Earth from the magnetotail, traveling close to the Sun-Earth line. The locations of Cluster and Rosetta are shown at the time the HFA and the Pc3 waves were observed.

with a pressure scaling based on $\mathrm{n}_{\mathrm{sw}}=8 \mathrm{~cm}^{-3}$ and $\left|\mathrm{v}_{\mathrm{sw}}\right|=$ $400 \mathrm{~km} \mathrm{~s}^{-1}$. It is found that before 21:06 UT, the magnetic field does not intercept the bow shock. At just before 21:06 UT, the magnetic field rotates so that from 21:0621:08 UT $\theta_{\mathrm{Bn}} \sim 60^{\circ}$. From 21:08-21:09 UT, $\theta_{\mathrm{Bn}} \sim 30^{\circ}$. During the HFA, the field mapping is evidently uncertain, and after the HFA (from 21:10 UT), magnetic field again does not intercept the shock. Consequently, the magnetic field is connected to the shock for a few minutes prior to the arrival of the TD, which provides the necessary source of particles for HFA formation. It should be noted that the field connects to oblique/quasi-perpendicular geometries which are the source of field aligned beams. During this connected interval, we do not see ULF waves, except for the packet associated with the HFA itself, where the wave periods are of the order of a few seconds.

[21] The location of Cluster is shown in Figure 2 together with the expected location of the magnetopause and bow shock based on the Farris et al. [1991] model. Cluster was located close to the shock (a few $\mathrm{R}_{\mathrm{E}}$ upstream), at [15, 2, 3] $\mathrm{R}_{\mathrm{E}}$ (GSE coordinates). To understand how the discontinuity interacted with the shock, Figure 3 shows the orientation of the underlying discontinuity in 3 dimensions and how it tracked across the bow shock. The discontinuity surface is defined as $\mathbf{n} .\left(\mathbf{r}-\mathbf{r}_{\mathbf{c}}\right)=0$ where $\mathbf{n}$ is the discontinuity normal and $\mathbf{r}_{\mathbf{c}}$ is the location of Cluster. Figure $3 \mathrm{a}$ shows the bow shock and discontinuity surface in 3 dimensions. The look direction is a few degrees off the Sun-Earth line, looking from the Sun. Cluster is represented as a blue dot, and the configuration is shown at 21:09 UT, when the discontinuity was observed by Cluster. It can be seen that the discontinuity intersects the bow shock along a line across the dusk flank of the shock.

[22] Figure 3b shows a 2-D plot of the evolution of the intersection line, projected in the $y-z$ GSE plane. Concentric circles show the location of the bow shock at $\mathrm{x}_{\mathrm{GSE}}=0,4$, 8 and $12 \mathrm{R}_{\mathrm{E}}$. The red line shows where the shock intersects the discontinuity at 21:09 UT at the time of observation. The location of Cluster is shown here as a black dot. Note that because of projection effects and the orientation of the underlying discontinuity, the black dot does not lie on the red line (this can be understood in the context of Figure 3a).

[23] The discontinuity is embedded in the solar wind, and so as the solar wind flows around the magnetosphere, the line of contact between the TD and the bow shock will change. To understand how the line of intersection moved with time, the location of the discontinuity was advected with the solar wind for a certain time interval $\Delta t$ and the new line of intersection calculated. In this case the discontinuity surface is given by $\mathbf{n} .\left(\mathbf{r}-\mathbf{r}_{\mathbf{c}}\right)-\left(\mathrm{v}_{\mathrm{sw}} \Delta \mathrm{t}\right) \mathbf{x}=0$. In Figure $3 \mathrm{~b}, \Delta \mathrm{t}=120 \mathrm{~s}$. Thus each line represents where the discontinuity intersected the bow shock at two minute intervals, starting $10 \mathrm{~min}$ before the observation and ending $10 \mathrm{~min}$ after. The motion of the discontinuity across the shock is shown by the arrow.

[24] It can be seen that the TD was interacting with a substantial fraction of the dusk flank shock surface several minutes before the HFA was observed by Cluster. Each line

Figure 1. Cluster magnetic field and plasma observations on 4 March 2005. (a-d) Magnetic field strength and components in the GSE coordinate system (e-i) ion bulk density, velocity, and temperature. Note that parallel and perpendicular temperatures are represented by dashed and solid lines respectively. Black, red, green, and blue are used to represent Cluster 1-4. Vertical lines mark the upstream and downstream intervals used in the analysis. 


\section{Cluster HFA 4 March 2005; Circles on shock at $x=0,4,8,12$ Re}

\section{Motion of discontinuity across shock (right to left) shown at 2 min resolution}
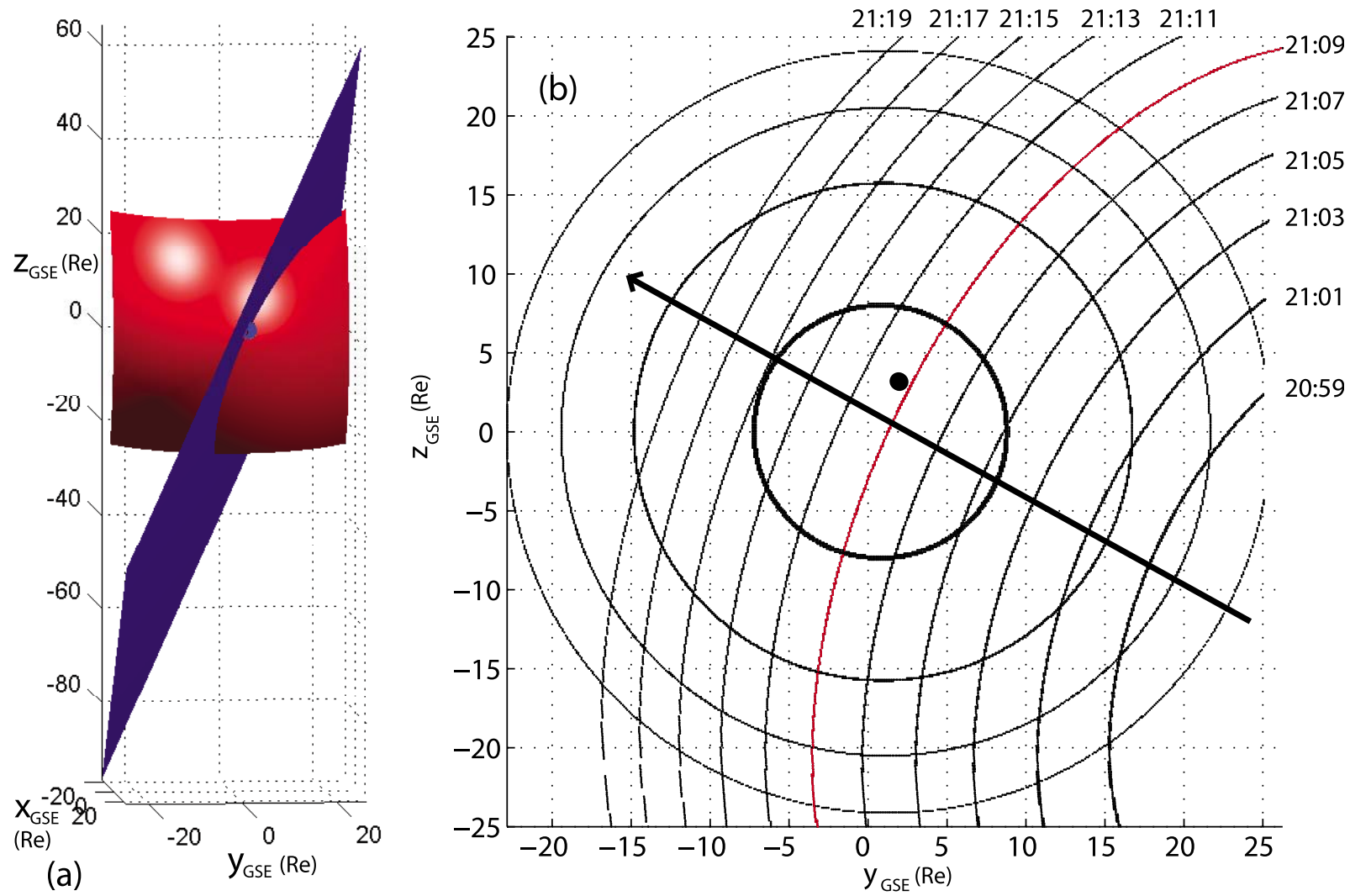

Figure 3. (a) Geometry of the interaction of the TD with the bow shock. (b) Motion of the intersection line between the bow shock and the TD as a function of time. The time at which each line is calculated is shown at the edge of the plot. The TD first touched the shock on the dusk flank below the ecliptic plane, and tracked across to the dawn flank, moving in the $-\mathrm{y}$ and $+\mathrm{z}$ GSE directions as indicated by the arrow.

of intersection is separated by approximately $2 \mathrm{R}_{\mathrm{E}}$, which given $\Delta \mathrm{t}=120 \mathrm{~s}$ corresponds to a speed of $\sim 100 \mathrm{~km} \mathrm{~s}^{-1}$, consistent with previous observations [Lucek et al., 2004].

\subsection{Rosetta and Ground-Based Pc3 Wave Observations}

[25] Figure 4 shows an overview of the Rosetta data; $12 \mathrm{~h}$ of data centered on closest approach are shown. In addition to the time of observation, $x$ axis labels show the location of the Rosetta spacecraft in the Geocentric Solar Magnetospheric (GSM) coordinate system. At the start of the interval, Rosetta was located at $\left[\begin{array}{lll}-18.6 & 2.0 & 0.4\end{array}\right] \mathrm{R}_{\mathrm{E}} \mathrm{GSM}$ and was moving Earthward. Following closest approach at 22:00 UT, Rosetta exited the dayside magnetosphere on the dawn flank $(y<0)$, below the ecliptic $(\mathrm{z}<0)$, crossing the magnetopause at 02:02 UT and the bow shock at 04:45 UT on 5 March 2005.

[26] Figure 5 shows the magnetic foot point of Rosetta, calculated using the Tsyganenko [1989] model. Lines of geographic latitude and longitude are dashed (note that $50^{\circ}$ $75^{\circ}$ is the range of latitude shown). Solid lines of invariant magnetic latitude between $60^{\circ}-75^{\circ}$ are also shown. The SunEarth line points vertically, with noon at the top of Figure 5. The foot point of Rosetta between 20:00 and 22:00 UT is shown as a thin black line. During this time the Rosetta foot point moved westward from midnight. Red crosses are used to indicate the location of the ground magnetometer stations used in the study.

[27] Figures $6 \mathrm{a}$ and $6 \mathrm{~b}$ show the $\mathrm{H}$ component of the magnetic field observed at PTRS in Alaska together with the wavelet transform of that component. There is a clear interval of Pc3 waves, beginning at 21:08 UT and lasting for several minutes until $21: 17: 30 \mathrm{UT}$. The frequency of the waves is $\sim 20 \mathrm{mHz}$, typical for Pc3 waves. Figures $6 \mathrm{c}$ and $6 \mathrm{~d}$ show the $\mathrm{H}$ component of the magnetic field observed at LER, the ground station closest to the foot point of Rosetta. Again, a clear interval of Pc3 waves is observed. Vertical black lines indicate the estimated onset and cessation of the wave activity, based on visual inspection of the time series. There is a delay between the onset of wave activity at PTRS and LER, which indicates that the disturbance was moving anti-sunward. LER was located at almost the same magnetic invariant latitude as PTRS, but the power of the observed waves was less, as might be expected for a signal propagating in the anti-sunward direction from the dayside magnetosphere. The duration of the waves at LER was $\sim 7.5 \mathrm{~min}$, less than at PTRS.

[28] Figures $6 \mathrm{e}$ and $6 \mathrm{f}$ show the $\mathrm{B}_{\mathrm{y}}$ component of the magnetic field measured by Rosetta. In addition to infor- 


\section{Rosetta March 2005}

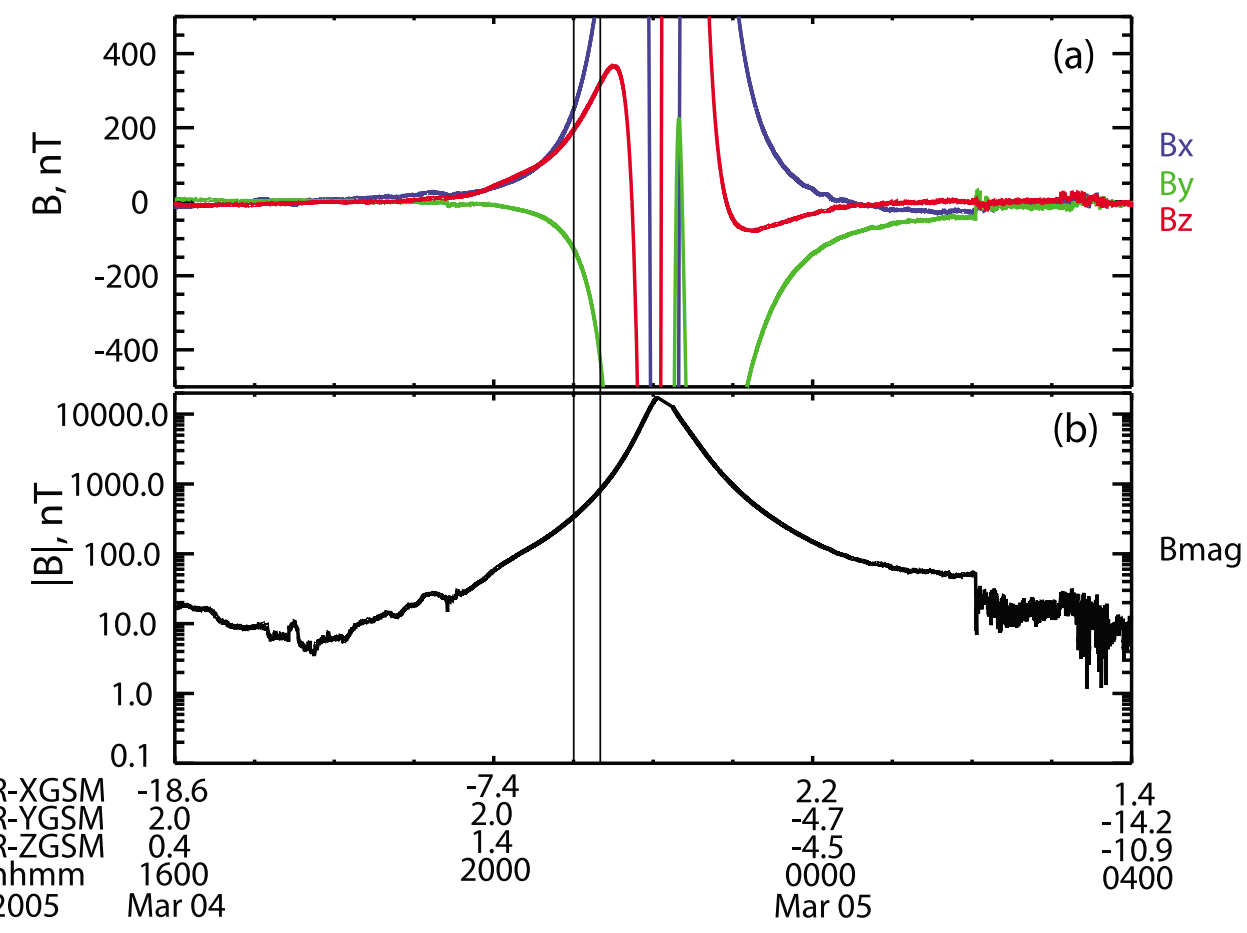

Figure 4. Overview of the Rosetta data (a) components of the magnetic field in the GSM coordinate system (b) magnetic field strength. The two black vertical lines mark the interval 2100-21:20 UT.

mation about the coordinates of the Rosetta spacecraft, the L shell of Rosetta is also shown based on the Tsyganenko [1989] magnetic field model. The model is parameterized according to the $\mathrm{Kp}$ index; on 4 March 2005 activity was very low and during the event $\mathrm{Kp}=0+$. In fact the mapping of Rosetta to the ionosphere at this time is relatively insensitive to the exact model used, and the spacecraft was located at $\mathrm{L}=5$. The geocentric distance from Rosetta to the Earth is indicated by $\mathrm{r} / \mathrm{Re}$; since Rosetta flew past the Earth above the geomagnetic equator, this distance is less than the L shell value. In Figure 5, the thick red line segment indicates where Rosetta was located when the interval of Pc3 waves was observed. The waves are less easily seen by eye in the time series since the background magnetic field is varying as the spacecraft moves toward closest approach. However, the wavelet transform of the data reveals the presence of the fluctuations, which are observed at the same time as those seen at LER. (Note the power scale in Figure $6 \mathrm{f}$ is different from Figures $6 \mathrm{~b}$ and $6 \mathrm{~d}$.)

[29] Figure 7 shows the $\mathrm{H}$ component of the magnetic field observed by the SAMNET magnetometers around the foot point of Rosetta in the interval 21:00-21:20 UT. The data have been bandpass filtered to leave fluctuations with periods between $10 \mathrm{~s}$ and $100 \mathrm{~s}$. This extends to slightly lower frequency than quoted frequencies for the Pc3 range (10 s to $45 \mathrm{~s})$, but captures the full frequency extent of the power based on the wavelet transforms shown in Figure 6. It can be seen that Pc3 pulsations were observed across the array.

\section{Discussion}

[30] The analysis of the Cluster data shows that an HFA was observed upstream of the bow shock as a result of a TD intersecting and interacting with the bow shock. It is thought that for the formation of an HFA to occur, two important requirements must be met: the first is that the convection electric field points into the discontinuity on an least one side, and the second is that the magnetic field must be connected to the shock on at least one side. It is found that

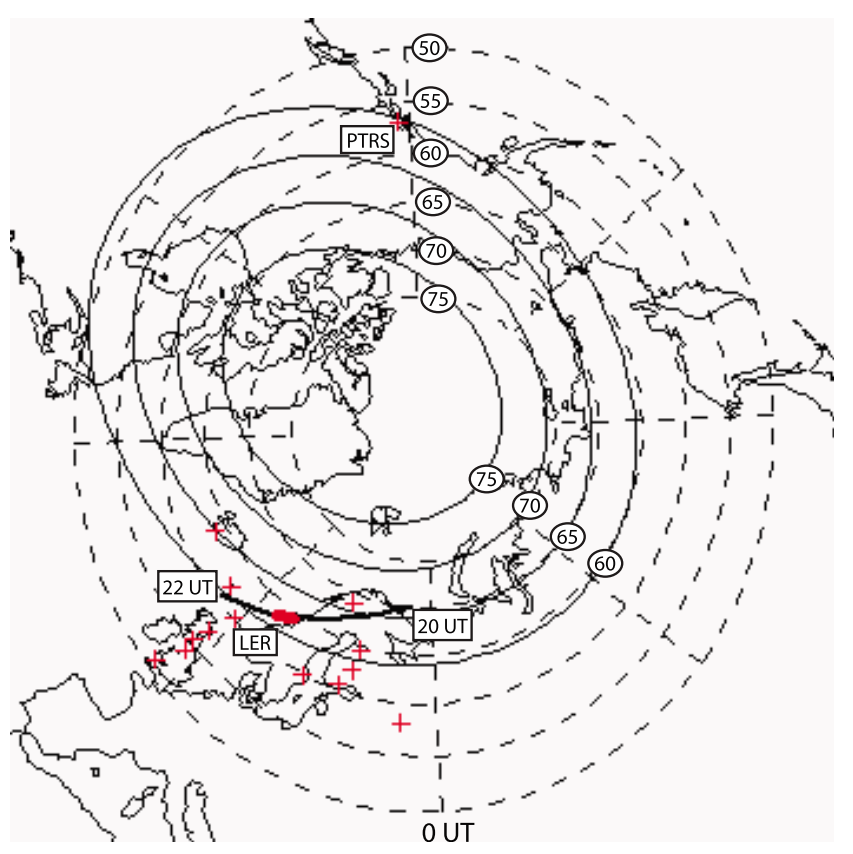

Figure 5. Magnetic foot point of Rosetta, shown together with the locations of the SAMNET ground magnetometers and the THEMIS GMAG station PTRS. 


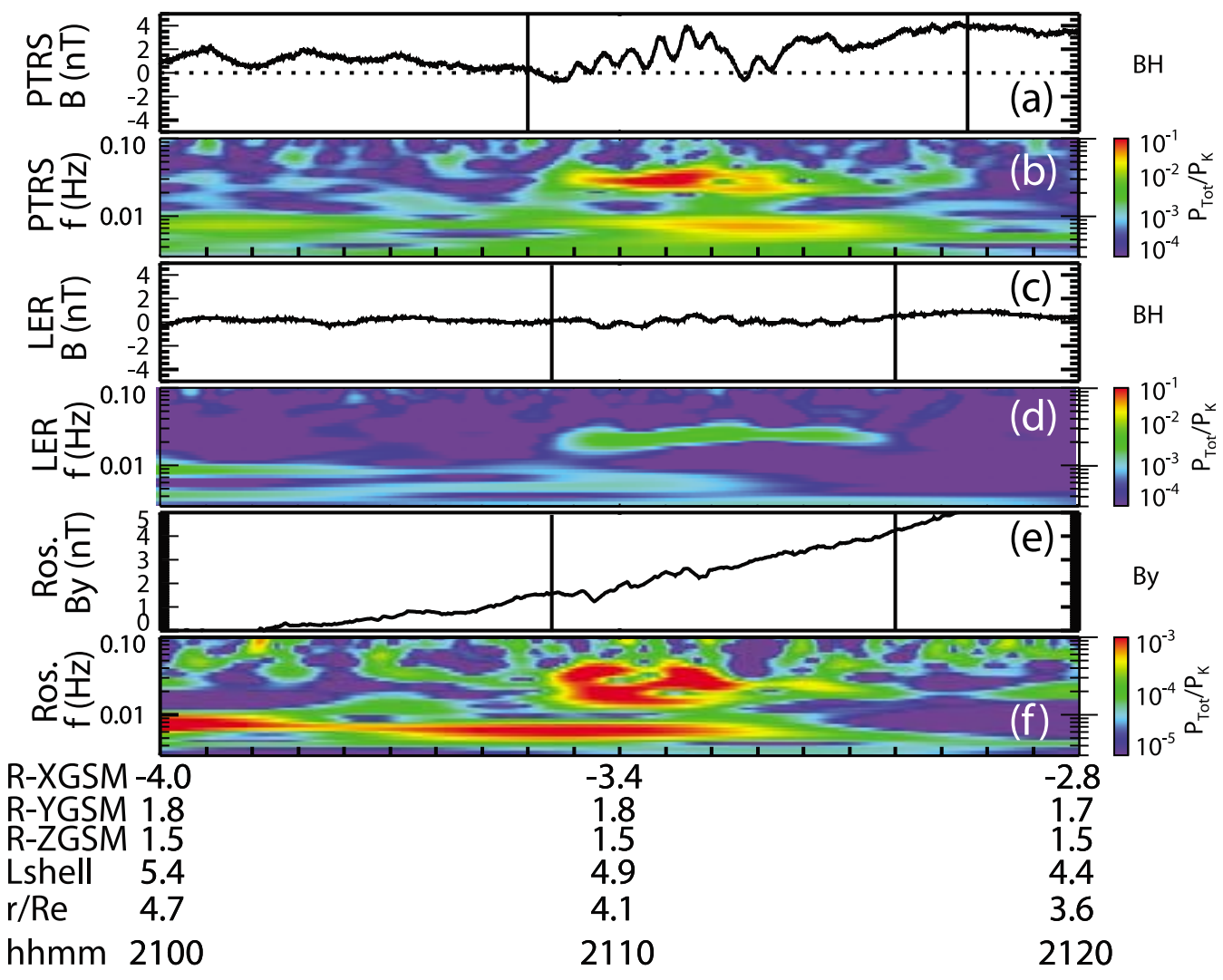

\section{Mar 04}

Figure 6. (a-b) Horizontal component of the magnetic field at THEMIS GMAG stations PTRS and wavelet transform. (c-d) H component of the magnetic field at LER and wavelet transform. (e-f) $\mathrm{B}_{\mathrm{y}}$ component of the magnetic field at Rosetta and wavelet transform.

the field is connected prior to the arrival of the TD, and that the convection electric field points inward on the trailing side. This lends confidence to the construction of the geometry and means that the TD acts like a wall approaching the shock, channeling the backstreaming particles along the discontinuity and generating the HFA. Furthermore, the angle between the TD normal and the solar wind flow is large, which ensures a slow traversal of the TD over the bow shock, providing sufficient time for the HFA to develop [Schwartz et al., 2000]. The geometry (shown in Figure 3) indicates that the TD underlying the HFA intersected the shock sunward of the terminator $(\mathrm{Y}>0)$ prior to the observation by Cluster, on the order of 10 min earlier (as indicated by the 20:59 line in Figure 3b).

[31] The HFA was observed between 21:08 and 21:10 UT by Cluster. The Pc3 waves were first observed at 21:08 UT by PTRS on the ground, and until 21:16 UT by all observers and until 21:18 UT by PTRS. We now consider the timeline of events. The transit time of signals from the magnetopause to the ground has been considered by Chi et al. [2001]. Assuming typical values for the properties of the plasmasphere and magnetosphere density, it is found that to propagate a signal across the magnetosphere takes of the order of $1 \mathrm{~min}$. The transit time across the magnetosheath from the bow shock to the magnetopause depends, to first order, on the magnetosheath flow speed. In the absence of in situ measurement this must be estimated. Given a solar wind speed of $400 \mathrm{~km} \mathrm{~s}^{-1}$, density $\sim 8 \mathrm{~cm}^{-3}$, temperature $\sim 0.1 \mathrm{MK}$, and magnetic field strength $\sim 3 \mathrm{nT}$, we calculate that $\mathrm{V}_{\text {Alfvén }}=$ $23 \mathrm{~km} \mathrm{~s}^{-1}, \mathrm{C}_{\text {sound }}=37 \mathrm{~km} \mathrm{~s}^{-1}$, and consequently that the solar wind magnetosonic mach number $M_{m s} \sim 9.2$ since $\mathrm{V}_{\mathrm{ms}}=$ $43.6 \mathrm{~km} \mathrm{~s}^{-1}$. Given the standard relationship for the velocity jump across a shock [e.g., Khan and Cowley, 1999],

$$
\frac{v_{\text {msheath }}}{v_{s w}}=\frac{(\gamma-1) M^{2}+2}{(\gamma+1) M^{2}},
$$

this implies that the magnetosheath velocity just downstream of the subsolar shock $v_{\text {msheath }} \sim 100 \mathrm{~km} \mathrm{~s}^{-1}$. The characteristic transit time $\Delta t$ for signals to be transmitted from the nose of the bow shock to the magnetopause is thus estimated to be [Clausen et al., 2009; Khan and Cowley, 1999]

$$
\Delta t=\frac{\Delta R}{v_{\text {msheath }}-v_{\text {mpause }}} \ln \left(\frac{v_{\text {msheath }}}{v_{\text {mpause }}}\right)
$$

Although exact calculations are evidently subject to uncertainty, if $\Delta \mathrm{R}=3 \mathrm{Re}$, and the speed of the magnetosheath flow just outside the magnetopause $v_{\text {mpause }}$ is taken as $20 \mathrm{~km} \mathrm{~s}^{-1}$ [Khan and Cowley, 1999] the transit time $\Delta \mathrm{t} \sim 6 \mathrm{~min}$. This provides an estimate of the fiducial magnetosheath transit time for the observed solar wind conditions, which is several times greater than the transit time through the magnetosphere. 


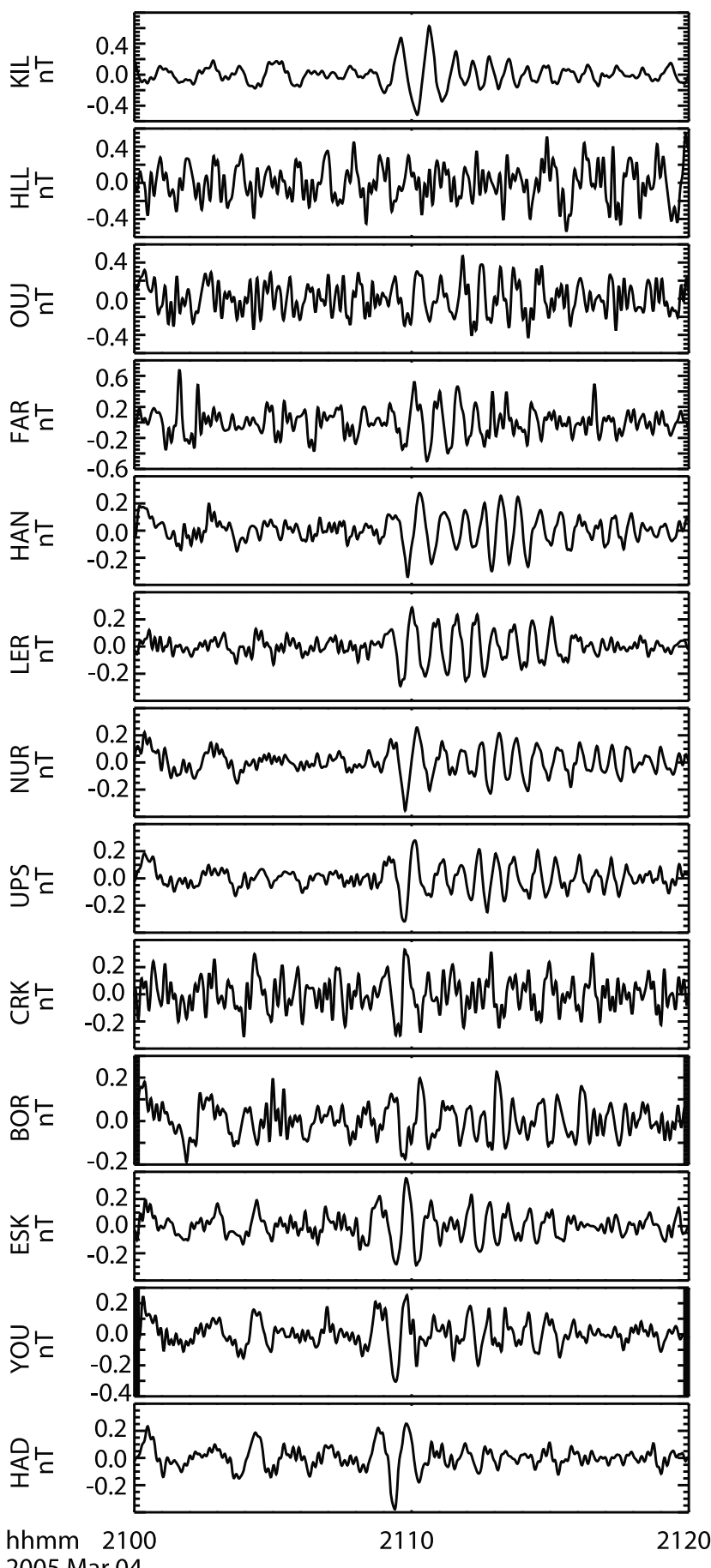

2005 Mar 04

Figure 7. Ground magnetometer data (horizontal component) ordered by magnetic latitude. The data have been bandpass filtered to show pulsations with periods of 10 $100 \mathrm{~s}$.

[32] However, the HFA disturbance developed on the flank of the shock. Away from the Sun-Earth line, the magnetosheath flow speed downstream of the shock is higher, but the distance from the shock to the vicinity of the magnetopause is also higher [Spreiter et al., 1966]. It is not trivial to determine a more precise estimate of the transit time, but it would appear that it should remain of the order of a few minutes. It is possible that dedicated global simulations may provide more insight into this problem in the future.

[33] If the total transit time from the HFA to the magnetopause and then from the magnetopause to the ground is $\sim 7 \mathrm{~min}$, this implies that the Pc3 waves first observed at 21:08 resulted from a disturbance at the shock that occurred at approximately 21:01 UT. Figure $3 \mathrm{~b}$ shows that at this time, the TD intersected the shock on the dusk flank approximately $10 \mathrm{R}_{\mathrm{E}}$ from the Sun-Earth line. As a consistency check, we note that not all magnetosheath flow lines approach the magnetopause; simple models of magnetosheath flow show that flow lines originating from the portion of the shock surface where $\mathrm{x}<8 \mathrm{R}_{\mathrm{E}}$ approach to within approximately $1 \mathrm{R}_{\mathrm{E}}$ of the magnetopause [Spreiter et al., 1966]. Thus the calculated delay would appear to be consistent with when the onset should be observed based on expected magnetosheath flow patterns and the speed with which the HFA moves across the shock. The Pc3 wave pulsations were observed until about 21:18 UT, corresponding to a few minutes after the HFA was observed by Cluster; by this time the HFA had transited to the dawn flank, away from the observation points at the nose of the magnetosphere. A timeline summarizing the observed events is shown in Figure 8.

[34] There are two possible scenarios for creating a limited interval of Pc3 wave activity. The first is that near the TD there is a region of solar wind that magnetically connects to the shock with a small magnetic field cone angle $\theta_{\mathrm{Bx}}$, surrounded by solar wind that does not connect efficiently (i.e., whose magnetic field cone angle $\theta_{\mathrm{Bx}}$ is large). This patch of connected solar wind contains ULF waves that are generated by backstreaming ions and this wave activity then couples through to the inner magnetosphere. This may be considered an adaptation of the "standard scenario," and does not specifically depend on the existence of the HFA itself.

[35] The ambient solar wind magnetic field strength was $\sim 3 \mathrm{nT}$ at this time, and so the predicted Pc3 frequency [Gul'elmi, 1974] is $6 \times 3=18 \mathrm{mHz}$. However, during this event no upstream waves were observed except for the packet associated with the leading edge of the HFA itself. The period of these waves was $<10 \mathrm{~s}$ and their duration was $\sim 1$ min; neither their period nor their duration corresponds to the observed Pc3 wave activity. Furthermore, the polarization of the waves in the spacecraft frame was righthanded, opposite to the usually observed left-handed polarization. Therefore Cluster, upstream of the bow shock and located close to the nose of the bow shock and magnetosphere did not observe a ULF wavefield that could be the source of the Pc3 waves as envisaged in the standard scenario for Pc3 wave generation. It could be argued that ULF waves were being generated in the region closer to the shock between Cluster and the shock surface, but as Figure 2 shows this is a small region since Cluster is only a few $R_{E}$ upstream. Given that the typical wavelength of $30 \mathrm{~s}$ period ULF waves is of the order of $1 \mathrm{R}_{\mathrm{E}}$ [Eastwood et al., 2005a], this distance would seem to be insufficient for the growth of such waves. Therefore we discount this scenario for this event. 


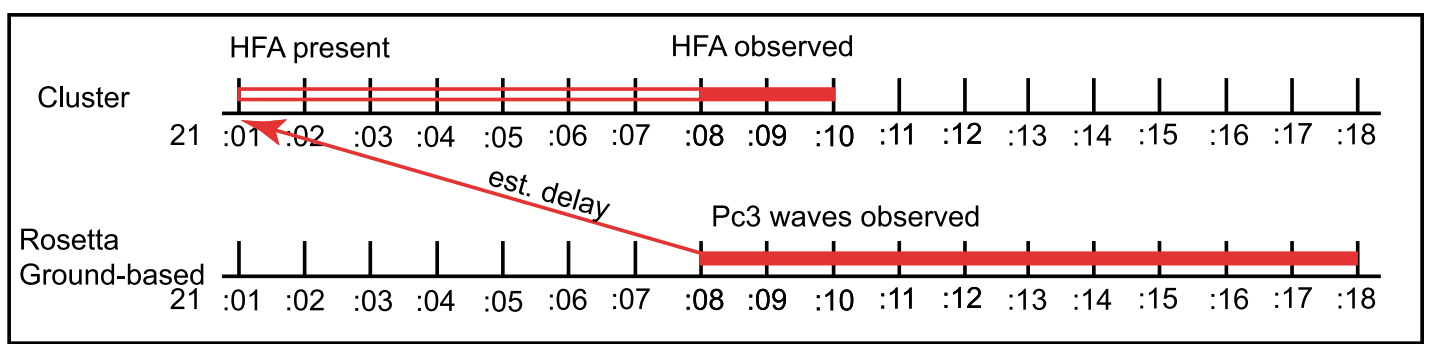

Figure 8. Timeline of the HFA event and observations.

[36] The second scenario is that the HFA creates a largescale disruption in the solar wind which transiently perturbs the magnetopause location. Previous observations show that HFAs can move the magnetopause boundary by several $R_{E}$ [e.g., Jacobsen et al., 2009]. As this disruption tracks along the bow shock and the subsequent perturbation, guided by the underlying TD, tracks along and interacts with the magnetopause, compressive waves are created and transmitted into the magnetosphere. As they propagate inward, they couple to field-line resonances (FLR), leading to the observed Pc3 wave signatures. The response of the magnetosphere to such an impulsive event has been considered in detail by Southwood and Kivelson [1990]. They find that most of the wave power from the perturbation is concentrated at $\mathrm{f}=\mathrm{k}_{\mathrm{y}} \mathrm{U}$ where $1 / \mathrm{k}_{\mathrm{y}}$ is the scale of the perturbation (which is treated as a pulse propagating in the $y$ direction over the magnetopause, similar to the data considered here), and $U$ is the speed with which the perturbation tracks over the magnetopause. The observed dominant Pc3 wave frequency is $\sim 20 \mathrm{mHz}$, and our calculations show that the HFA tracks over the bow shock at $\sim 100 \mathrm{~km} \mathrm{~s}^{-1}$, as discussed at the end of section 3.1. This implies that the scale size of the HFA perturbation on the magnetopause is $U / f \sim 1 R_{E}$. Although we do not have direct measurements of the magnetopause in this event, this scale is consistent with the typical transverse scale size of HFAs. The overall scale and duration of the HFA observed at Cluster during this event is fairly average since the central core of the HFA passes over Cluster in a few tens of seconds. This picture therefore suggests that HFAs should naturally inject wave power in the Pc3 frequency range, and that the Pc3 waves should last as long as the HFA is tracking along the bow shock over the nose of the magnetosphere. We note that at the SAMNET locations, the FLR frequencies are close to, but somewhat lower than, the observed wave frequency [Wild et al., 2005]. The onset of the Pc3 wave activity is thus consistent with the timing and motion of the HFA's interaction with the bow shock, and the frequency of the observed waves is related to the scale size of the HFA and the pressure pulse that it generates as it moves along the magnetopause.

\section{Conclusions}

[37] As such, we conclude in this event that the interval of Pc3 waves observed by Rosetta and ground based magnetometers was not due to the interval of magnetically connected solar wind that occurred prior to the HFA, but to the HFA itself, and the disruption on the magnetopause that the HFA is presumed to have caused. The timing of the
HFA is consistent with the onset of the Pc3 waves at Rosetta and on the ground. No upstream waves of the appropriate frequency were observed upstream by Cluster just upstream of the nose of the shock prior to their observation in the inner magnetosphere and on the ground. The exact nature of the small packet of waves observed on the leading edge of the HFA remains unclear, and a separate study is required to understand this aspect of the observations. However, the exact mechanism by which the HFA perturbed the magnetopause and subsequently generated Pc3 waves cannot be explored within the context of this satellite conjunction, because no magnetopause observations were available.

[38] This result is complementary to previously published observations showing that intervals of appropriately oriented IMF cause Pc3 wave activity on the ground [e.g., Clausen et al., 2009]. However, it demonstrates that in connecting upstream activity to the ground observations, one must also be careful to include the effects of the bow shock, which can be highly nonlinear, as manifest in this instance by the HFA. It may explain, at least in part, the second population of $\mathrm{Pc} 3$ waves, whose frequency does not depend on $|\mathrm{B}|_{\mathrm{sw}}$. In particular, the frequency of HFA-generated Pc3 waves would appear to depend on the transverse scale size of the HFA, which tends to be of the order of a few Earth radii; this may explain why the frequency of the second population is insensitive to $\mid \mathrm{BI}_{\mathrm{sw}}$. It should be noted that in previous HFA studies Pc3 waves were not reported, and so more work is required to understand exactly why this is the case. Since the data presented here come from a unique conjunction, future investigations examining other data sets (e.g., Cluster, THEMIS, and the planned NSF CINEMA Cubesat) are required to completely elucidate the exact mechanism by which the dramatic, large-scale, nonlinear disruption caused by HFAs at the bow shock results in Pc3 pulsations in the magnetosphere and on the ground.

[39] Acknowledgments. Cluster and Rosetta data analysis at Imperial College London is supported by a Science and Technology Facilities Council (STFC) Rolling Grant. The contribution of the IGeP team was financially by the German Bundesministerium für Wirtschaft und Technologie and the Deutsches Zentrum für Luft-und Raumfahrt under the contracts $50 \mathrm{QP}$ 1001 and 50 OC 0901. This study has made use of the Cluster Active Archive. The Sub-Auroral Magnetometer Network (SAMNET) is operated by the Space Plasma Environment and Radio Science group in the Department of Physics at Lancaster University. We acknowledge NASA contract NAS5-02099 and V. Angelopoulos for use of data from the THEMIS Mission. Specifically, S. Mende and C. T. Russell for use of the GMAG data. J.P.E. holds an STFC Advanced Fellowship at ICL.

[40] Masaki Fujimoto thanks the reviewers for their assistance in evaluating this paper. 


\section{References}

Balogh, A., et al. (2001), The Cluster magnetic field investigation: Overview of inflight performance and initial results, Ann. Geophys., 19, 1207-1217, doi:10.5194/angeo-19-1207-2001

Burch, J. L., R. Goldstein, T. E. Cravens, W. C. Gibson, R. N. Lundin, C. J. Pollock, J. D. Winningham, and D. T. Young (2007), RPC-IES: The ion and electron sensor of the Rosetta plasma consortium, Space Sci. Rev., 128, 697-712, doi:10.1007/s11214-006-9002-4.

Burgess, D. (1995), Foreshock-shock interaction at collisionless quasiparallel shocks, Adv. Space Res., 15(8-9), 159-169, doi:10.1016/ 0273-1177(94)00098-L.

Burgess, D. (1997), What do we really know about upstream waves?, $A d v$ Space Res., 20(4-5), 673-682, doi:10.1016/S0273-1177(97)00455-9.

Carr, C., et al. (2007), RPC: The Rosetta Plasma Consortium, Space Sci. Rev., 128, 629-647, doi:10.1007/s11214-006-9136-4.

Chi, P. J., and C. T. Russell (1998), Phase skipping and Poynting flux of continuous pulsations, J. Geophys. Res., 103(A12), 29,479-29,491, doi:10.1029/98JA02101

Chi, P. J., et al. (2001), Propagation of the preliminary reverse impulse of sudden commencements to low latitudes, J. Geophys. Res., 106(A9), 18,857-18,864, doi:10.1029/2001JA900071.

Clausen, L. B. N., T. K. Yeoman, R. C. Fear, R. Behlke, E. A. Lucek, and M. J. Engebretson (2009), First simultaneous measurements of waves generated at the bow shock in the solar wind, the magnetosphere and on the ground, Ann. Geophys., 27, 357-371, doi:10.5194/angeo-27357-2009.

Davison, S. J., and D. Orr (1989), A global pulsation event with conjugate study, Planet. Space Sci., 37, 253-267, doi:10.1016/0032-0633(89) 90023-8

Eastwood, J. P., A. Balogh, E. A. Lucek, C. Mazelle, and I. Dandouras (2003), On the existence of Alfvén waves in the terrestrial foreshock, Ann. Geophys., 21, 1457-1465, doi:10.5194/angeo-21-1457-2003.

Eastwood, J. P., A. Balogh, E. A. Lucek, C. Mazelle, and I. Dandouras (2005a), Quasi-monochromatic ULF foreshock waves as observed by the four-spacecraft Cluster mission: 1. Statistical properties, J. Geophys. Res., 110, A11219, doi:10.1029/2004JA010617.

Eastwood, J. P., E. A. Lucek, C. Mazelle, K. Meziane, Y. Narita, J. S. Pickett, and R. A. Treumann (2005b), The foreshock, Space Sci. Rev., 118, 41-94, doi:10.1007/s11214-005-3824-3.

Eastwood, J. P., et al. (2008), THEMIS observations of a hot flow anomaly: Solar wind, magnetosheath and ground-based measurements, Geophys. Res. Lett., 35, L17S03, doi:10.1029/2008GL033475.

Escoubet, C. P., M. Fehringer, and M. L. Goldstein (2001), The Cluster mission, Ann. Geophys., 19, 1197-1200, doi:10.5194/angeo-19-11972001.

Farris, M. H., S. M. Petrinec, and C. T. Russell (1991), The thickness of the magnetosheath: Constraints on the polytropic index, Geophys. Res. Lett. 18(10), 1821-1824, doi:10.1029/91GL02090.

Fillingim, M. O., J. P. Eastwood, G. K. Parks, V. Angelopoulos, I. R. Mann, S. B. Mende, and A. T. Weatherwax (2011), Polar UVI and THEMIS GMAG observations of the ionospheric response to a hot flow anomaly, J. Atmos. Sol. Terr. Phys., 73, 137-145, doi:10.1016/j.jastp.2010.03.001.

Fuselier, S. A. (1994), Suprathermal ions upstream and downstream from the Earth's bow shock, in Solar Wind Sources of Magnetospheric Ultralow-Frequency Waves, Geophys. Monogr. Ser., vol. 81, edited by M. J. Engebretson, K. Takahashi, and M. Scholer, pp. 107-119, AGU, Washington, D. C.

Fuselier, S. A. (1995), Ion distributions in the Earth's foreshock upstream from the bow shock, Adv. Space Res., 15(8-9), 43-52, doi:10.1016 0273-1177(94)00083-D.

Glassmeier, K.-H. (1992), Traveling magnetospheric convection twinvortices - Observations and theory, Ann. Geophys., 10, 547-565.

Glassmeier, K.-H., H. Boehnhardt, D. Koschny, E. Kührt, and I. Richter (2007a), The Rosetta Mission: Flying towards the origin of the solar system, Space Sci. Rev., 128, 1-21, doi:10.1007/s11214-006-9140-8.

Glassmeier, K.-H., et al. (2007b), RPC-MAG: The fluxgate magnetometer in the Rosetta Plasma Consortium, Space Sci. Rev., 128, 649-670, doi:10.1007/s11214-006-9114-X.

Gul'elmi, A. V. (1974), Diagnostics of the magnetosphere and interplanetary medium by means of pulsations, Space Sci. Rev., 16, 331-345, doi:10.1007/BF00171562.

Hughes, W. J. (1994), Magnetospheric ULF waves: A tutorial with historical perspective, in Solar Wind Sources of Magnetospheric Ultralow Frequency Waves, Geophys. Monogr. Ser., vol. 81, edited by M. J. Engebretson, K. Takahashi, and M. Scholer, pp. 1-11, AGU, Washington, D. C.

Jacobsen, K. S., et al. (2009), THEMIS observations of extreme magnetopause motion caused by a hot flow anomaly, J. Geophys. Res., 114, A08210, doi:10.1029/2008JA013873.
Khan, H., and S. W. H. Cowley (1999), Observations of the response time of high-latitude ionospheric convection to variations in the interplanetary magnetic field using EISCAT and IMP-8 data, Ann. Geophys., 17, 1306-1335, doi:10.1007/s00585-999-1306-8.

Knetter, T., F. M. Neubauer, T. S. Horbury, and A. Balogh (2004), Four-point discontinuity observations using Cluster magnetic field data: A statistical survey, J. Geophys. Res., 109, A06102, doi:10.1029/2003JA010099.

Lanzerotti, L. J., L. C. Lee, C. G. Maclennan, A. Wolfe, and L. V. Medford (1986), Possible evidence of flux transfer events in the polar ionosphere, Geophys. Res. Lett., 13(11), 1089-1092, doi:10.1029/GL013i011p01089.

Le, G., and C. T. Russell (1994), The morphology of ULF waves in the Earth's foreshock, in Solar Wind Sources of Magnetospheric UltralowFrequency Waves, Geophys. Monogr. Ser., vol. 81, edited by M. J. Engebretson, K. Takahashi, and M. Scholer, pp. 87-98, AGU, Washington, D. C.

Lucek, E. A., T. S. Horbury, A. Balogh, I. Dandouras, and H. Rème (2004) Cluster observations of hot flow anomalies, J. Geophys. Res., 109, A06207, doi:10.1029/2003JA010016.

Lühr, H., A. Aylward, S. C. Bucher, A. Pajunpää, K. Pajunpää, T. Holmboe, and S. M. Zalewski (1998), Westward moving dynamic substorm features observed with the IMAGE magnetometer network and other ground-based instruments, Ann. Geophys., 16, 425-440, doi:10.1007/ s00585-998-0425-y

Mende, S. B., S. E. Harris, H. U. Frey, V. Angelopoulos, C. T. Russell, E. Donovan, B. Jackel, M. Greffen, and L. M. Peticolas (2008), The THEMIS array of ground-based observatories for the study of auroral substorms, Space Sci. Rev., 141, 357-387, doi:10.1007/s11214-008-9380-x.

Odera, T. J. (1986), Solar wind controlled pulsations: A review, Rev. Geophys., 24(1), 55-74, doi:10.1029/RG024i001p00055.

Omidi, N., and D. G. Sibeck (2007), Formation of hot flow anomalies and solitary shocks, J. Geophys. Res., 112, A01203, doi:10.1029/ 2006JA011663.

Peticolas, L. M., et al. (2008), The Time History of Events and Macroscale Interactions during Substorms (THEMIS) Education and Outreach (E/PO) Program, Space Sci. Rev., 141, 557-583, doi:10.1007/s11214008-9458-5.

Rème, H., et al. (2001), First multispacecraft ion measurements in and near the Earth's magnetosphere with the identical Cluster ion spectrometry (CIS) experiment, Ann. Geophys., 19, 1303-1354, doi:10.5194/ angeo-19-1303-2001

Russell, C. T., P. J. Chi, D. J. Dearborn, Y. S. Ge, B. Kuo-Tiong, J. D. Means, D. R. Pierce, K. M. Rowe, and R. C. Snare (2008), THEMIS ground-based magnetometers, Space Sci. Rev., 141, 389-412, doi:10.1007/s11214-008-9337-0.

Schwartz, S. J. (1995), Hot flow anomalies near the Earth's bow shock, Adv. Space Res., 15(8-9), 107-116, doi:10.1016/0273-1177(94)00092-F.

Schwartz, S. J., G. Paschmann, N. Sckopke, T. M. Bauer, M. W. Dunlop, A. N. Fazakerley, and M. F. Thomsen (2000), Conditions for the formation of hot flow anomalies at the Earth's bow shock, J. Geophys. Res. 105(A6), 12,639-12,650, doi:10.1029/1999JA000320.

Shields, D. W., et al. (2003), Multistation studies of the simultaneous occurrence rate of Pc 3 micropulsations and magnetic impulsive events, J. Geophys. Res., 108(A6), 1225, doi:10.1029/2002JA009397.

Sibeck, D. G., et al. (1999), Comprehensive study of the magnetospheric response to a hot flow anomaly, J. Geophys. Res., 104(A3), 4577-4593, doi:10.1029/1998JA900021.

Sitar, R. J., J. B. Baker, C. R. Clauer, A. J. Ridley, J. A. Cummock, V. O Papitashvili, J. Spann, M. J. Brittnacher, and G. K. Parks (1998), Multiinstrument analysis of the ionospheric signatures of a hot flow anomaly occurring on July 24, 1996, J. Geophys. Res., 103(A10), 23,357-23,372, doi:10.1029/98JA01916.

Slawinski, R., D. Venkatesan, A. Wolfe, L. J. Lanzerotti, and C. G. Maclennan (1988), Transmission of solar wind hydromagnetic energy into the terrestrial magnetosphere, Geophys. Res. Lett., 15(11), 1275-1278, doi:10.1029/GL015i011p01275.

Southwood, D. J., and M. Kivelson (1990), The magnetohydrodynamic response of the magnetospheric cavity to changes in solar wind pressure, J. Geophys. Res., 95(A3), 2301-2309, doi:10.1029/JA095iA03p02301.

Spreiter, J. R., A. L. Summers, and A. Y. Alksne (1966), Hydromagnetic flow around the magnetosphere, Planet. Space Sci., 14, 223-253, doi:10.1016/0032-0633(66)90124-3.

Takahashi, K., R. L. McPherron, and T. Terasawa (1984), Dependence of the spectrum of Pc3-4 pulsations on the interplanetary magnetic field, J. Geophys. Res., 89(A5), 2770-2780, doi:10.1029/JA089iA05p02770. Thomsen, M. F., V. A. Thomas, D. Winske, J. T. Gosling, M. H. Farris, and C. T. Russell (1993), Observational test of hot flow anomaly formation by the interaction of a magnetic discontinuity with the bow shock, J. Geophys. Res., 98(A9), 15,319-15,330, doi:10.1029/93JA00792. 
Troitskaya, V. A., A. V. Gul'elmi, O. V. Bolshakova, E. T. Matveyeva, and R. V. Schepetnov (1972), Indices of geomagnetic pulsations, Planet. Space Sci., 20, 849-858, doi:10.1016/0032-0633(72)90170-5.

Tsyganenko, N. A. (1989), A magnetospheric magnetic field model with a warped tail current sheet, Planet. Space Sci., 37, 5-20, doi:10.1016 0032-0633(89)90066-4.

Weatherwax, A. T., H. B. Vo, T. J. Rosenberg, S. B. Mende, H. U. Frey, L. J. Lanzerotti, and C. G. Maclennan (1999), A dayside ionospheric absorption perturbation in response to a large deformation of the magnetopause, Geophys. Res. Lett., 26(4), 517-520, doi:10.1029/ 1999GL900017.

Wild, J. A., T. K. Yeoman, and C. L. Waters (2005), Revised time-of-flight calculations for high-latitude geomagnetic pulsations using a realistic magnetospheric magnetic field model, J. Geophys. Res., 110, A11206, doi:10.1029/2004JA010964.
Zesta, E., W. J. Hughes, and M. J. Engebretson (2002), A statistical study of traveling convection vortices using the Magnetometer Array for Cusp and Cleft Studies, J. Geophys. Res., 107(A10), 1317, doi:10.1029/ 1999JA000386.

C. M. Carr, J. P. Eastwood, T. S. Horbury, and S. J. Schwartz, Blackett Laboratory, Imperial College London, Prince Consort Road, London SW7 2AZ, UK. (jonathan.eastwood@imperial.ac.uk)

K.-H. Glassmeier, C. Koenders, F. Plaschke, and I. Richter, IGEP, Technische Universität Braunschweig, Mendelssohnstr. 3, Braunschweig D-38106, Germany.

J. A. Wild, Space Plasma Environment and Radio Science Group Department of Physics, Lancaster University, Lancaster LA1 4YB, UK. 